\title{
Utility of a pediatric fast magnetic resonance imaging protocol as surveillance scanning for traumatic brain injury
}

\author{
Chelsea Shope, BS, ${ }^{1}$ Mohammed Alshareef, MD, ${ }^{2}$ Thomas Larrew, MD, ${ }^{2}$ Christopher Bolling, MD, ${ }^{3}$ \\ Justin Reagan, MD, ${ }^{3}$ Milad Yazdani, MD, ${ }^{3}$ Maria Spampinato, MD, ${ }^{3}$ and Ramin Eskandari, MD, MS ${ }^{2}$ \\ ${ }^{1}$ College of Medicine, Medical University of South Carolina; and Departments of ${ }^{2}$ Neurosurgery and ${ }^{3}$ Radiology, Medical \\ University of South Carolina, Charleston, South Carolina
}

\begin{abstract}
OBJECTIVE Traumatic brain injury (TBI) is a prevalent pediatric pathology in the modern emergency department. Computed tomography (CT) is utilized for detection of TBI and can result in cumulatively high radiation exposure. Recently, a fast brain magnetic resonance imaging (fbMRI) protocol has been employed for rapid imaging of hydrocephalus in pediatric patients. The authors investigate the utility of a modified trauma-focused fbMRI (t-fbMRI) protocol as an alternative to surveillance $\mathrm{CT}$ in the setting of acute TBI in pediatric patients, thus reducing radiation exposure while improving diagnostic yield.
\end{abstract}

METHODS A retrospective review was performed at the authors' institution for all pediatric patients who had undergone t-fbMRI within 72 hours of an initial CT scan, using a 1.5- or 3-T MR scanner for trauma indications. Forty patients met the study inclusion criteria. The authors performed a comparison of findings on the reads of CT and fbMRI, and a boardcertified neuroradiologist conducted an independent review of both modalities.

RESULTS T-fbMRI outperformed CT in specificity, sensitivity, and negative predictive value for all injury pathologies measured, except for skull fractures. T-fbMRI demonstrated a sensitivity of $100 \%$ in the detection of extraaxial bleed, intraventricular hemorrhage, and subarachnoid hemorrhage and had a sensitivity of $78 \%$ or greater for epidural hematoma, subdural hematoma, and intraparenchymal hemorrhage. T-fbMRI yielded a specificity of $100 \%$ for all types of intracranial hemorrhages, with a corresponding negative predictive value that exceeded that for CT.

CONCLUSIONS In pediatric populations, the t-fbMRI protocol provides a valid alternative to $\mathrm{CT}$ in the surveillance of $\mathrm{TBI}$ and intracranial hemorrhage. Although not as sensitive in the detection of isolated skull fractures, t-fbMRI can be used to monitor pathologies implicated in TBI patients while minimizing radiation exposure from traditional surveillance imaging.

https://thejns.org/doi/abs/10.3171/2020.8.PEDS20496

KEYWORDS traumatic brain injury; pediatric trauma; magnetic resonance imaging; computed tomography; radiation; intracranial hemorrhage; surveillance imaging

$\mathrm{E}$ VERY year, nearly 630,000 emergency department visits are attributed to children under 18 years of age suffering from traumatic brain injury (TBI). ${ }^{1-3}$ Prompt accurate evaluation of these patients is paramount to triage those with urgent surgical needs versus those requiring conservative observation. ${ }^{4,5}$ Over the last 30 years, computed tomography (CT) has been a rapid, presumably accurate source of imaging when a child requires head and/or brain evaluation because of its speed, availability, and diagnostic ability to rule out severe acute intracranial injury. ${ }^{6}$ Although the modality is clinically useful, the emission of ionizing radiation increases the risk of brain neoplasm and leukemia, especially in children and adolescents who are more radiosensitive. ${ }^{7}$ Brenner et al. ${ }^{8}$ found that each noncontrast head CT is estimated to contribute a lifetime cancer mortality risk of $0.7 \%$ in a 1 -year-old child, which is an order of magnitude higher than in an adult. Moreover, children with TBI may undergo multiple CT scans, creating an additive effect. ${ }^{9,10}$

Magnetic resonance imaging (MRI) utilization in pe-

ABBREVIATIONS ADC = apparent diffusion coefficient; $\mathrm{CT}=$ computed tomography; $\mathrm{DWI}=$ diffusion-weighted imaging; $\mathrm{EDH}=$ epidural hematoma; $\mathrm{GCS}=\mathrm{Glasgow}$ Coma Scale; GRE = gradient echo; HASTE = half-Fourier acquisition single-shot turbo spin echo; IPH = intraparenchymal hemorrhage; IVH = intraventricular hemorrhage; MRI = magnetic resonance imaging; NPV = negative predictive value; $\mathrm{PPV}=$ positive predictive value; $\mathrm{SAH}=$ subarachnoid hemorrhage; $\mathrm{SDH}=$ subdural hematoma; $\mathrm{TBI}=$ traumatic brain injury; $\mathrm{t}$-fbMRI = trauma-focused fast brain MRI.

SUBMITTED June 7, 2020. ACCEPTED August 19, 2020

INCLUDE WHEN CITING Published online February 5, 2021; DOI: 10.3171/2020.8.PEDS20496. 

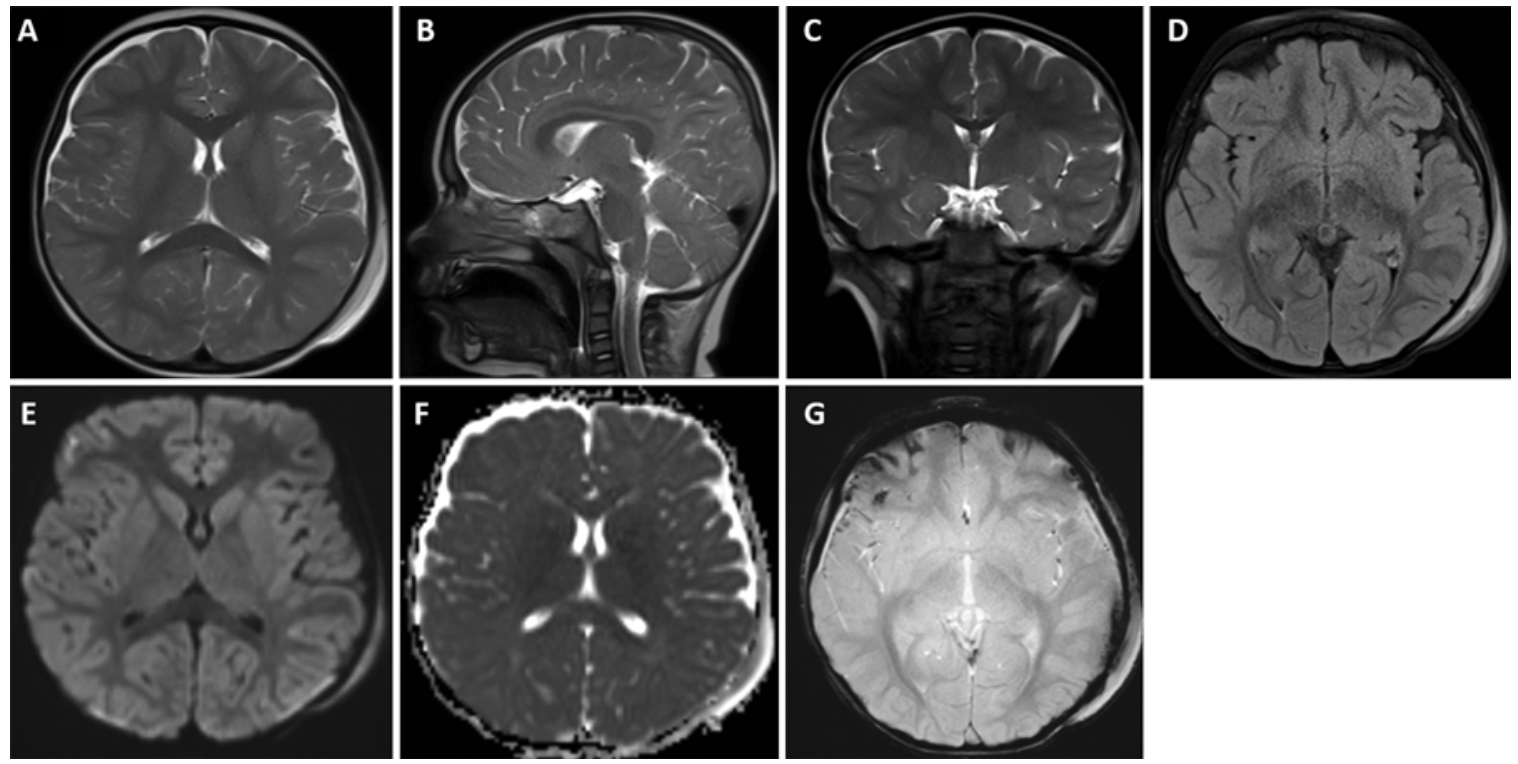

FIG. 1. Selected sequences utilizing the t-fbMRI protocol. The seven sequences were as follows: T2-weighted HASTE in the axial $(A)$, sagittal (B), and coronal (C) planes; T2-weighted FLAIR in the axial plane (D); DWI trace in the axial plane (E); ADC in the axial plane (F); and GRE in the axial plane $(\mathbf{G})$.

diatric acute TBI has historically been avoided because of untimely access, the need for patients to be motionless, and the paucity of literature supporting its ability to rule out clinically significant intracranial injuries. ${ }^{11-13}$ Patient motion requiring sedation or intubation is likely the largest hurdle to MRI use, especially with very young patients, as anesthesia time in infants and toddlers is now being linked to adverse events and mild cognitive injury. ${ }^{14-17}$

Recently, trauma-focused fast brain MRI (t-fbMRI) protocols have demonstrated validity for surveillance and follow-up imaging of TBI in patients with head trauma, including at our institution. ${ }^{18,19}$ Lindberg and colleagues ${ }^{20}$ published a landmark paper in Pediatrics in 2019 in which they followed a cohort of almost 300 children, comparing the feasibility and accuracy of fast MRI with that of CT in the surveillance of TBI. Our study not only attempts to validate Lindberg and colleagues' findings but also reports on an independently designed t-fbMRI sequence, the breakdown of MRI accuracy for all intracranial hemorrhages independently, and the negative predictive values for CT versus MRI by using independent, blinded evaluations. The objective of this paper was to show that the accuracy of $t$-fbMRI as a surveillance tool for TBI is noninferior to traditional CT scanning, making it a safe and effective alternative imaging modality.

\section{Methods \\ Data Collection}

An institutional review board-approved retrospective chart review was performed for all pediatric patients who had undergone t-fbMRI at the Medical University of South Carolina, a level I pediatric trauma center, between June 2018 and June 2019. Inclusion criteria were an age younger than 18 years, presentation with traumatic head injury requiring a head CT scan without contrast, and a follow-up t-fbMRI within 72 hours. The time frame was determined using the start time on the initial CT and the start time on the initial t-fbMRI. Patients who had sustained nonaccidental trauma were excluded. Of the 172 patients identified, 40 met our inclusion criteria.

Data pertaining to demographics, mechanism of trauma, presenting Glasgow Coma Scale (GCS) score, time between $\mathrm{CT}$ and $\mathrm{t}$-fbMRI, relevant time information related to t-fbMRI, radiological findings on CT and t-fbMRI, and required surgical interventions were collected. TfbMRI was performed for further prognostication of TBI following acute trauma.

\section{Imaging Protocol}

At our institution, the t-fbMRI protocol includes seven sets of image sequences: T2-weighted half-Fourier acquisition single-shot turbo spin echo (HASTE) imaging in the axial, sagittal, and coronal planes; diffusion-weighted imaging (DWI); apparent diffusion coefficient (ADC) in the axial plane; T2-weighted FLAIR in the axial plane; and gradient echo (GRE) in the axial plane (Fig. 1). Imaging was performed on a 1.5- or 3-T MR scanner (Siemens Healthcare). The procedure takes 7 minutes to complete. No sedation or patient restraints were used for imaging.

\section{Imaging Interpretation}

Radiology reports from CT and t-fbMRI studies were reviewed for the presence or absence of the following terms: skull fracture, subarachnoid hemorrhage (SAH), epidural hematoma (EDH), subdural hematoma (SDH), extraaxial bleed (either SDH or EDH), and intraventricular or intraparenchymal hemorrhage (IVH or IPH). Each study was finalized by a board-certified neuroradiologist. Retrospective review of CT and t-fbMRI scans was performed by a separate board-certified neuroradiologist 
(M.S.) using AGFA Impax. Images were assigned a score of either 0 or 1 based on image quality. A score of 1 was assigned to studies that were of acceptable image quality and answered the clinical question. Studies that did not meet these criteria were assigned a score of 0 . CT and tfbMRI studies were evaluated blindly and independent of one another.

\section{Repeat Review for Rigor}

In order to adequately assess the presence of skull fractures, t-fbMRI scans underwent two separate and independent reviews, without CT for comparison. A boardcertified neuroradiologist performed an additional review of CT scans that had been read as positive for hemorrhage on initial presentation but were read as negative for hemorrhage with follow-up t-fbMRI. A subsequent review of all CT images was performed alongside the followup t-fbMRI for comparison to determine whether blood products were truly present on initial CT.

Statistical analysis was run in duplicate. Initial statistics used CT findings as the gold standard for true positive and negative when comparing t-fbMRI results. A second statistical analysis (corrected) allowed comparison of the t-fbMRI with CT to confirm true-positive and true-negative findings. Each analysis determined sensitivity, specificity, and positive and negative predictive values (PPV and NPV) for t-fbMRI findings compared with CT in each category of injury. A $p$ value $<0.05$ was considered significant.

\section{Statistical Evaluation}

All statistical analyses were performed using IBM SPSS (IBM Corp.) to compare CT and t-fbMRI radiological findings. We utilized Cohen's kappa coefficient $(\kappa)$ for agreement between CT and t-fbMRI for the presence of skull fracture and all categories of intracranial hemorrhage (SAH, SDH, EDH, IVH, and IPH). Agreement measures were defined as follows: $\kappa \leq 0.20$, poor agreement; $\kappa=0.21-0.40$, fair agreement; $\kappa=0.41-0.60$, moderate agreement; $\kappa=0.61-0.80$, good agreement; $\kappa=0.81-1$, very good agreement.

\section{Results}

\section{Participant Characteristics}

During the study period, 40 patients met the inclusion criteria. Twenty-five patients were female and 15 were male. The average age was 6.7 years (range 14.6 days-17 years), and the average presenting GCS score was 12.5 (range 3-15). The most common mechanism of trauma was a fall, followed by high-speed-impact accidents such as motor vehicle collisions. Patients warranted admission because of either a moderate (GCS score 9-13) or a severe (GCS score 3-8) TBI or positive findings on CT (Table 1). The average time between initial CT and followup t-fbMRI was 7.64 hours (range 1.38-30.67 hours). Of the 40 study participants, 18 were transfers from outside hospitals where they had received their original CT scans. The average time from the t-fbMRI order to the neuroradiology report created was 1.73 hours (range 0.27-11.47 hours).
TABLE 1. Demographic and imaging characteristics in 40 patients with TBI

\begin{tabular}{lc}
\hline \multicolumn{1}{c}{ Variable } & Value \\
\hline Gender & \\
\hline Female & $25(63)$ \\
\hline Male & $15(38)$ \\
\hline Age in yrs & $6.7 \pm 5.8$ \\
\hline Mechanism of trauma & $22(55)$ \\
\hline Fall & $17(43)$ \\
\hline High-speed impact & $1(3)$ \\
\hline Crush injury & $12.5 \pm 3.9$ \\
\hline GCS score & $28(70)$ \\
\hline 14-15, mild & $6(15)$ \\
\hline $9-13$, moderate & $6(15)$ \\
\hline $3-8$, severe & $7.64 \pm 6.3$ \\
\hline Time btwn CT \& MRI in hrs & $1.73 \pm 2.2$ \\
\hline Time from MRI completion to report created in hrs & $5(13)$ \\
\hline Neurosurgical intervention $(n=5)$ & $5(100)$ \\
\hline SAH & $3(60)$ \\
\hline EDH & $2(40)$ \\
\hline SDH & $4(80)$ \\
\hline IPH &
\end{tabular}

Values are expressed as number (\%) or mean \pm standard deviation.

\section{Comparative Radiological Findings}

Cohen's kappa coefficient demonstrated good agreement between CT and t-fbMRI when comparing SAH $(\kappa$ $=0.63)$ and skull fracture $(\kappa=0.74)$ identified on $\mathrm{t}$-fbMRI. The same method demonstrated fair agreement between $\mathrm{CT}$ and $\mathrm{t}$-fbMRI for SDH $(\kappa=0.48)$ and IVH $(\kappa=0.48)$. Cohen's kappa coefficient showed poor agreement between CT and t-fbMRI for EDH $(\kappa=0.22)$, extraaxial bleed $(\kappa=0.33)$, and IPH $(\kappa=0.34$; Table 2$)$.

\section{Sensitivity}

When CT and t-fbMRI were evaluated as stand-alone studies, t-fbMRI outperformed CT for all hemorrhage types. The sensitivity of t-fbMRI for detecting an extraaxial bleed, IVH, and SAH (100\% for each category) was substantially better than the sensitivity of CT $(38 \%, 33 \%$, and $86 \%$, respectively; Table 3). T-fbMRI detected SDH with a sensitivity of $93 \%$ versus CT with $57 \%$, IPH with a sensitivity of $85 \%$ versus $54 \%$, and $\mathrm{EDH}$ with a sensitivity of $78 \%$ versus $11 \%$. Initial statistical analysis suggested a high sensitivity of t-fbMRI in skull fracture detection (81\%). When these data were reevaluated using the blinded protocol detailed above without $\mathrm{CT}$ comparison available, the sensitivity of $\mathrm{t}$-fbMRI in detecting skull fracture dropped to $56 \%$, considerably lower than that of CT $(100 \%)$.

\section{Specificity}

T-fbMRI also outperformed CT in each category for specificity, with six of the seven categories demonstrating a specificity of $100 \%$ : SAH (vs $81 \%$ with CT), SDH (vs $92 \%$ ), IPH (vs 93\%), EDH (vs 100\%), IVH (vs 100\%), and 
TABLE 2. Radiological findings using CT as the gold standard

\begin{tabular}{|c|c|c|c|c|c|c|c|c|}
\hline $\begin{array}{l}\text { Radiological } \\
\text { Finding }\end{array}$ & $\begin{array}{c}\text { Positive Finding } \\
\text { on CT (no.) }\end{array}$ & $\begin{array}{l}\text { Positive Finding } \\
\text { on fbMRI (no.) }\end{array}$ & $\begin{array}{l}\text { Negative Finding } \\
\text { on CT (no.) }\end{array}$ & $\begin{array}{l}\text { Negative Finding } \\
\text { on fbMRI (no.) }\end{array}$ & $\begin{array}{l}\text { Correlation } \\
\qquad(\kappa)\end{array}$ & $p$ Value & $\begin{array}{l}\text { Sensitivity } \\
\text { of fbMRI }\end{array}$ & $\begin{array}{l}\text { Specificity } \\
\text { of fbMRI }\end{array}$ \\
\hline $\mathrm{SAH}$ & 17 & 14 & 23 & 26 & 0.63 & $<0.0001$ & $71 \%$ & $91 \%$ \\
\hline Extraaxial bleed & 7 & 10 & 33 & 30 & 0.33 & $<0.031$ & $57 \%$ & $82 \%$ \\
\hline $\mathrm{EDH}$ & 1 & 7 & 39 & 33 & 0.22 & $<0.028$ & $100 \%$ & $85 \%$ \\
\hline $\mathrm{SDH}$ & 11 & 14 & 29 & 26 & 0.48 & $<0.002$ & $73 \%$ & $79 \%$ \\
\hline IVH & 1 & 3 & 39 & 37 & 0.48 & $<0.0001$ & $100 \%$ & $95 \%$ \\
\hline $\mathrm{IPH}$ & 9 & 11 & 31 & 29 & 0.34 & $<0.032$ & $56 \%$ & $81 \%$ \\
\hline Skull fracture (initial) & 27 & 22 & 13 & 18 & 0.74 & $<0.0001$ & $81 \%$ & $100 \%$ \\
\hline Skull fracture (blinded) & 27 & 15 & 13 & 25 & 0.45 & $<0.0001$ & $56 \%$ & $100 \%$ \\
\hline
\end{tabular}

Cohen's kappa (к): $\leq 0.20$, poor agreement; $0.21-0.40$, fair agreement; $0.41-0.60$, moderate agreement; $0.61-0.80$, good agreement; $0.81-1$, very good agreement.

skull fracture (vs 100\%). When t-fbMRI was evaluated without CT comparison, the specificity for detecting skull fracture dropped to $85 \%$.

\section{Positive Predictive Value}

$\mathrm{t}$-fbMRI demonstrated higher PPV than CT across all hemorrhage categories, with a value of $80 \%$ or higher. The PPV of t-fbMRI was $100 \%$ for IPH (vs $87 \%$ with CT), SDH (vs 88\%), EDH (vs 100\%), and IVH (vs 100\%). The initial t-fbMRI PPV for skull fracture was $100 \%$ (vs $100 \%$ with CT), but it was $93 \%$ when corrected. The PPV of $\mathrm{t}$-fbMRI was $80 \%$ for SAH (vs $74 \%$ with CT; Table 4).

\section{Negative Predictive Value}

T-fbMRI demonstrated a higher NPV than CT across all hemorrhage categories, with a value of $93 \%$ or higher. Pathologies with an NPV of 100\% included SAH (vs with 93\% CT), IVH (vs 95\% with CT), and extraaxial bleed (vs $86 \%$ with CT). The NPV of t-fbMRI was $96 \%$ for SDH (vs $81 \%$ with CT), $94 \%$ for EDH (vs 79\%), and $93 \%$ for IPH (vs $82 \%$ ). Again, CT had a higher NPV for the detection of skull fracture (100\%) than t-fbMRI (72\% initially, corrected to $52 \%$; Table 4 ).

\section{Discussion}

TBI is a prevalent pathology in the modern pediatric

TABLE 3. Comparison of sensitivity and specificity of MRI and CT following the revised gold-standard method

\begin{tabular}{lrrrr}
\hline \multirow{1}{*}{ Variable } & $\begin{array}{c}\text { CT } \\
\text { Sensitivity }\end{array}$ & $\begin{array}{c}\text { t-fbMRI } \\
\text { Sensitivity }\end{array}$ & $\begin{array}{c}\text { CT } \\
\text { Specificity }\end{array}$ & $\begin{array}{c}\text { t-fbMRI } \\
\text { Specificity }\end{array}$ \\
\hline SAH & $86 \%$ & $100 \%$ & $81 \%$ & $100 \%$ \\
\hline Extraaxial bleed & $38 \%$ & $100 \%$ & $88 \%$ & $94 \%$ \\
\hline EDH & $11 \%$ & $78 \%$ & $100 \%$ & $100 \%$ \\
\hline SDH & $57 \%$ & $93 \%$ & $92 \%$ & $100 \%$ \\
\hline IVH & $33 \%$ & $100 \%$ & $100 \%$ & $100 \%$ \\
\hline IPH & $54 \%$ & $85 \%$ & $93 \%$ & $100 \%$ \\
\hline Skull fracture (initial) & $100 \%$ & $81 \%$ & $100 \%$ & $100 \%$ \\
\hline $\begin{array}{l}\text { Corrected skull } \\
\text { fracture (blinded) }\end{array}$ & $100 \%$ & $56 \%$ & $100 \%$ & $85 \%$ \\
\hline
\end{tabular}

emergency department, most commonly detected using $\mathrm{CT}$. Given accumulating evidence for the negative effects of ionizing radiation exposure in children attributed to CT, MRI has become a modality of interest for evaluation of TBI. In the present study, t-fbMRI was performed as the follow-up study after initial CT imaging in children with TBI. In our series, participants did not require intubation, sedation, or restraints for completion of $t-f b M R I$. The ability to accurately identify all intracranial hemorrhages was higher with t-fbMRI than with standard noncontrast head $\mathrm{CT}$, but not in identifying skull fracture. Findings missed on follow up t-fbMRI were clinically nonsignificant in our study. Similarly, multiple prior studies have demonstrated linear, nondisplaced fractures to be clinically nonsignificant $^{21}$ and, unless associated with additional underlying intracranial injury, to carry minimal risk for related complications. $^{6,22-25}$

In this study, we initially designated the original CT scan as the gold standard and thus used $\kappa$ as a comparison evaluation for $\mathrm{t}-\mathrm{fbMRI}$ against the $\mathrm{CT}$ scan, as reported in Lindberg and colleagues' 2019 Pediatrics landmark paper, which observed greater than $90 \%$ sensitivity and specificity for TBI using fbMRI. ${ }^{20}$ Using their method of comparison, we found $\mathrm{t}-\mathrm{fbMRI}$ to have a sensitivity of $100 \%$ for IVH and EDH and greater than $79 \%$ specificity for each category of hemorrhage (Table 2). This high specificity allows clinicians to rule in only those patients requiring admission, avoiding unnecessary hospitalizations.

TABLE 4. Comparison of PPV and NPV of MRI and CT following the revised gold-standard method

\begin{tabular}{lrrrr}
\hline \multicolumn{1}{c}{ Variable } & CT & t-fbMRI & CT & t-fbMRI \\
& PPV & PPV & NPV & NPV \\
\hline SAH & $74 \%$ & $80 \%$ & $93 \%$ & $100 \%$ \\
\hline Extraaxial bleed & $67 \%$ & $80 \%$ & $86 \%$ & $100 \%$ \\
\hline EDH & $100 \%$ & $100 \%$ & $79 \%$ & $94 \%$ \\
\hline SDH & $88 \%$ & $100 \%$ & $81 \%$ & $96 \%$ \\
\hline IVH & $100 \%$ & $100 \%$ & $95 \%$ & $100 \%$ \\
\hline IPH & $87 \%$ & $100 \%$ & $82 \%$ & $93 \%$ \\
\hline Skull fracture (initial) & $100 \%$ & $100 \%$ & $100 \%$ & $72 \%$ \\
\hline Corrected skull fracture (blinded) & & $93 \%$ & & $52 \%$ \\
\hline
\end{tabular}



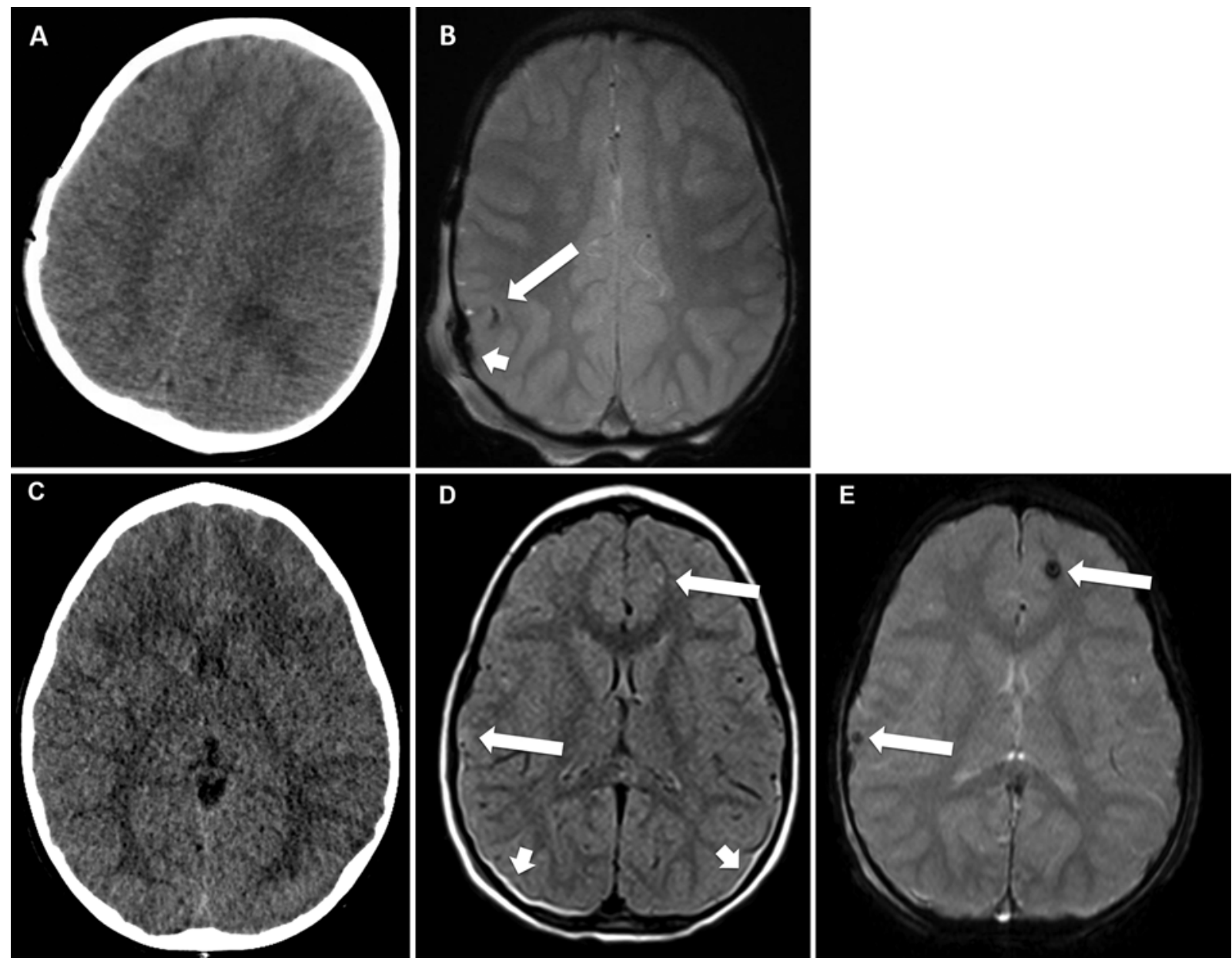

FIG. 2. Example of intracranial hemorrhages identified on surveillance trauma MRI but not readily identifiable on an initial CT scan. Images (A, B) obtained in a 3-year-old female with TBI following crush injury. The CT (A) reveals SAH and skull fracture, prompting hospital admission and a surveillance scan. Surveillance MRI, an axial GRE sequence (B), reveals SAH, as well as EDH (short arrow) and IPH (long arrow) that had not been previously demonstrated on CT. Images (C-E) obtained in a 2-year-old female who presented with a severe TBI following a high-speed motor vehicle collision. The CT (C) reveals a scattered SAH with no other intracranial bleeds. Surveillance MRI, an axial FLAIR sequence (D), reveals areas of hyperintensity concerning for IPH (Iong white arrows, D and E) as well as small occipital SDHs (short arrows) not previously demonstrated on CT. IPH is confirmed on the accompanying GRE sequence $(\mathrm{E})$.

However, CT imaging does have a propensity to miss intracranial pathology (Fig. 2). We would therefore challenge the current notion that CT may be the gold standard for identification of all intracranial hemorrhage pathologies from acute TBI. Given that CT is also a fallible tool, we obtained independent reviews of both $\mathrm{CT}$ and $\mathrm{t}-\mathrm{fbMRI}$ by nonblinded neuroradiologists after all blinded evaluations were complete. This second round of statistical analysis helped to verify whether initial findings on CT were accurate or reflected artifact (bone, motion, or partial volume averaging). Following these secondary reviews, t-fbMRI had an even higher sensitivity and specificity for the detection of all types of hemorrhage measured.

The average time between CT and t-fbMRI for our study was only $7.64 \pm 6.3$ hours. Such a short interval provides a unique opportunity for a close comparison of t-fbMRI and CT, an advantage over a prior study with an average of 27.5 hours between scans. ${ }^{26}$ The average time between ordering t-fbMRI and the created neuroradiology report was 1.73 hours. We realize that if this modality is to be used as a primary imaging method, the time between ordering and the final report of the findings needs to be dramatically decreased. Given that our t-fbMRI was used as follow-up imaging for patients who are typically within the pediatric intensive care unit or the stepdown unit, the time between ordering and report is quite fast.

The goal of our t-fbMRI protocol is a nonradiating, nonsedating, and reliable surveillance tool following TBI. Three-plane T2-weighted images demonstrate gross pathology. FLAIR visualizes edema and inflammation, whereas GRE is useful for identifying blood products. ${ }^{27}$ DWI and ADC are compared to evaluate for acute infarcts. Notably, previous studies have lacked sequences most sensitive for blood products, that is, GRE and SWI. ${ }^{28-32}$ In our study, the combination of FLAIR and GRE allowed for the detection of small microhemorrhages, whereas 
GRE helped to visualize SDH and EDH, as have been observed in previous studies..$^{10,33,34}$ The literature studies that included GRE and DWI showed higher MRI sensitivity rates than those that did not. ${ }^{20,35,36}$ Multisequence MRI performed as a fast scan is a relatively new technological advance that overcomes previous limitations of detecting blood products in sulci, cisterns, and ventricles and along the convexity where neighboring cerebrospinal fluid can mask its presence.

\section{Conclusions}

T-fbMRI compared to CT for children with TBI offers higher sensitivity, specificity, PPV, and NPV for all categories of intracranial hemorrhage. Our findings help validate and expand on prior studies while demonstrating the speed with which t-fbMRI studies can be obtained. Whether t-fbMRI can replace the initial trauma protocol head CT for children in the emergency department is still controversial.

We acknowledge that our study has several limitations, including a small sample size, non-immediate follow-up imaging, large variation in participant age, and inconsistent CT scans given the large proportion of patients transferring to our hospital given its status as a tertiary care level I pediatric trauma center. Yet, we are confident that our findings, along with those of others vying to improve imaging for children suffering from TBI, will collectively push the bar in a direction in which these limitations will become obsolete.

\section{References}

1. Faul M, Xu L, Wald MM, Coronado VG. Traumatic Brain Injury in the United States: Emergency Department Visits, Hospitalizations and Deaths 2002-2006. Centers for Disease Control and Prevention, National Center for Injury Prevention and Control; 2010:75.

2. Schneier AJ, Shields BJ, Hostetler SG, et al. Incidence of pediatric traumatic brain injury and associated hospital resource utilization in the United States. Pediatrics. 2006; 118(2):483-492.

3. Stanley RM, Bonsu BK, Zhao W, et al. US estimates of hospitalized children with severe traumatic brain injury: implications for clinical trials. Pediatrics. 2012;129(1):e24-e30.

4. Vavilala MS, King MA, Yang JT, et al. The Pediatric Guideline Adherence and Outcomes (PEGASUS) programme in severe traumatic brain injury: a single-centre hybrid implementation and effectiveness study. Lancet Child Adolesc Health. 2019;3(1):23-34.

5. Stevens RD, Shoykhet M, Cadena R. Emergency neurological life support: intracranial hypertension and herniation. Neurocrit Care. 2015;23(2)(suppl 2):S76-S82.

6. Pickering A, Harnan S, Fitzgerald P, et al. Clinical decision rules for children with minor head injury: a systematic review. Arch Dis Child. 2011;96(5):414-421.

7. Pearce MS, Salotti JA, Little MP, et al. Radiation exposure from CT scans in childhood and subsequent risk of leukaemia and brain tumours: a retrospective cohort study. Lancet. 2012;380(9840):499-505.

8. Brenner D, Elliston C, Hall E, Berdon W. Estimated risks of radiation-induced fatal cancer from pediatric CT. AJR Am J Roentgenol. 2001;176(2):289-296.

9. Young AMH, Guilfoyle MR, Donnelly J, et al. Multimodality neuromonitoring in severe pediatric traumatic brain injury. Pediatr Res. 2018;83(1-1):41-49.
10. Greenberg JK, Jeffe DB, Carpenter CR, et al. North American survey on the post-neuroimaging management of children with mild head injuries. J Neurosurg Pediatr. 2018; 23(2):227-235.

11. Wintermark M, Sanelli PC, Anzai Y, et al. Imaging evidence and recommendations for traumatic brain injury: conventional neuroimaging techniques. J Am Coll Radiol. 2015;12(2): e1-e14.

12. Filippi C, Sanelli P. Pediatric traumatic brain injury: common data elements to inform diagnosis, neuroimaging, and outcome metrics. J Pediatr Neuroradiol. 2016;5(1):32-37.

13. Argyropoulou MI, Alexiou GA, Xydis VG, et al. Pediatric minor head injury imaging practices: results from an ESPR survey. Neuroradiology. 2020;62(2):251-255.

14. Mendoza D, Kadom N, Palasis S, et al. Use of conventional and advanced MRI techniques in accidental pediatric traumatic brain injury. J Pediatr Neuroradiol. 2016;5(1):20-25.

15. Malviya S, Voepel-Lewis T, Eldevik OP, et al. Sedation and general anaesthesia in children undergoing MRI and CT: adverse events and outcomes. Br J Anaesth. 2000;84(6):743-748.

16. Flick RP, Katusic SK, Colligan RC, et al. Cognitive and behavioral outcomes after early exposure to anesthesia and surgery. Pediatrics. 2011;128(5):e1053-e1061.

17. O'Leary JD, Janus M, Duku E, et al. Influence of surgical procedures and general anesthesia on child development before primary school entry among matched sibling pairs. JAMA Pediatr. 2019;173(1):29-36.

18. Mehta $\mathrm{H}$, Acharya J, Mohan AL, et al. Minimizing radiation exposure in evaluation of pediatric head trauma: use of rapid MR imaging. AJNR Am J Neuroradiol. 2016;37(1):11-18.

19. Kabakus IM, Spampinato MV, Knipfing M, et al. Fast brain magnetic resonance imaging with half-Fourier acquisition with single-shot turbo spin echo sequence in detection of intracranial hemorrhage and skull fracture in general pediatric patients: preliminary results. Pediatr Emerg Care. 2019.

20. Lindberg DM, Stence NV, Grubenhoff JA, et al. Feasibility and accuracy of fast MRI versus CT for traumatic brain injury in young children. Pediatrics. 2019;144(4):e20190419.

21. Greenes DS, Schutzman SA. Clinical significance of scalp abnormalities in asymptomatic head-injured infants. Pediatr Emerg Care. 2001;17(2):88-92.

22. Kuppermann N, Holmes JF, Dayan PS, et al. Identification of children at very low risk of clinically-important brain injuries after head trauma: a prospective cohort study. Lancet. 2009; 374(9696):1160-1170.

23. Osmond MH, Klassen TP, Wells GA, et al. CATCH: a clinical decision rule for the use of computed tomography in children with minor head injury. CMAJ. 2010;182(4):341-348.

24. Dunning J, Daly JP, Lomas JP, et al. Derivation of the children's head injury algorithm for the prediction of important clinical events decision rule for head injury in children. Arch Dis Child. 2006;91(11):885-891.

25. Lyttle MD, Crowe L, Oakley E, et al. Comparing CATCH, CHALICE and PECARN clinical decision rules for paediatric head injuries. Emerg Med J. 2012;29(10):785-794.

26. Sheridan DC, Newgard CD, Selden NR, et al. QuickBrain MRI for the detection of acute pediatric traumatic brain injury. J Neurosurg Pediatr. 2017;19(2):259-264.

27. Currie S, Saleem N, Straiton JA, et al. Imaging assessment of traumatic brain injury. Postgrad Med J. 2016;92(1083):41-50.

28. Young JY, Duhaime AC, Caruso PA, Rincon SP. Comparison of non-sedated brain MRI and CT for the detection of acute traumatic injury in children 6 years of age or less. Emerg Radiol. 2016;23(4):325-331.

29. Dremmen MHG, Wagner MW, Bosemani T, et al. Does the addition of a "black bone" sequence to a fast multisequence trauma MR protocol allow MRI to replace CT after traumatic brain injury in children? AJNR Am J Neuroradiol. 2017; 38(11):2187-2192. 
30. Flom L, Fromkin J, Panigrahy A, et al. Development of a screening MRI for infants at risk for abusive head trauma. Pediatr Radiol. 2016;46(4):519-526.

31. Kralik SF, Yasrebi M, Supakul N, et al. Diagnostic performance of ultrafast brain MRI for evaluation of abusive head trauma. AJNR Am J Neuroradiol. 2017;38(4):807-813.

32. Ryan ME, Jaju A, Ciolino JD, Alden T. Rapid MRI evaluation of acute intracranial hemorrhage in pediatric head trauma. Neuroradiology. 2016;58(8):793-799.

33. Wang ML, Li WB. Cognitive impairment after traumatic brain injury: the role of MRI and possible pathological basis. J Neurol Sci. 2016;370:244-250.

34. Roguski M, Morel B, Sweeney M, et al. Magnetic resonance imaging as an alternative to computed tomography in select patients with traumatic brain injury: a retrospective comparison. J Neurosurg Pediatr. 2015;15(5):529-534.

35. Atzema C, Mower WR, Hoffman JR, et al. Prevalence and prognosis of traumatic intraventricular hemorrhage in patients with blunt head trauma. J Trauma. 2006;60(5):10101017.

36. Rocchi G, Caroli E, Raco A, et al. Traumatic epidural hematoma in children. J Child Neurol. 2005;20(7):569-572.

\section{Disclosures}

The authors report no conflict of interest concerning the materials or methods used in this study or the findings specified in this paper.

\section{Author Contributions}

Conception and design: Alshareef, Shope, Eskandari. Acquisition of data: Alshareef, Shope, Bolling, Reagan, Yazdani, Spampinato. Analysis and interpretation of data: Alshareef, Shope, Bolling, Reagan, Yazdani, Spampinato. Drafting the article: Alshareef, Shope, Larrew, Eskandari. Critically revising the article: Alshareef, Shope, Larrew, Yazdani, Spampinato, Eskandari. Reviewed submitted version of manuscript: all authors. Approved the final version of the manuscript on behalf of all authors: Alshareef. Statistical analysis: Alshareef. Study supervision: Alshareef, Eskandari.

\section{Supplemental Information}

\section{Previous Presentations}

This work was presented as a poster presentation at the Annual Meeting of the AANS/CNS Section on Pediatric Neurological Surgery held in Scottsdale, Arizona, on December 5-8, 2019.

\section{Correspondence}

Ramin Eskandari: Medical University of South Carolina, Charleston,SC.eskandar@musc.edu. 